\title{
Actividad Fitotóxica de un Extracto N-Hexano ObTenido de La Corteza de DRIMYS Winteri SObRe Cuatro Especies de Malezas ${ }^{1}$
}

\author{
Phytotoxic Activity of N-Hexane Extract Obtained From Drimys Winteri Bark on Four Weeds
}

\author{
ZAPATA, N. ${ }^{2}$, VARGAS, M. $^{2}$ y MEDINA, P. ${ }^{3}$
}

\begin{abstract}
RESUMEN - E1 objetivo de esta investigación fue determinar el efecto fitotóxico de un extracto obtenido con n-hexano de la corteza de Drimys winteri sobre la germinación y el crecimiento de Convolvulus arvensis, Setaria pumila, Daucus carota y Cichorium intybus. El efecto fitotóxico del extracto sobre la germinación de las malezas se determinó mediante bioensayos en placas de Petri y la aplicación del extracto en el medio de germinación en concentraciones de 100 a $1.000 \mathrm{mg} \mathrm{L}^{-1}$. En bioensayos en macetas, se determinó el efecto fitotóxico del extracto sobre la emergencia y crecimiento de las plántulas de malezas, en los cuales se aplicó el extracto mezclado con el sustrato de cultivo en concentraciones de 500 a $4.000 \mathrm{mg} \mathrm{kg}^{-1}$. También se determinó el efecto fitotóxico del extracto asperjado sobre plantas de malezas en concentraciones de 800 a $6.400 \mathrm{mg} \mathrm{L}^{-1}$. En los ensayos de germinación, el extracto retrasó la germinación y redujo el crecimiento de tallo y radicula de todas las malezas, el porcentaje de germinación disminuyó en todas las malezas, excepto en C. arvensis. La aplicación del extracto al sustrato de cultivo disminuyó y retrasó la emergencia de todas las malezas. Las plantas tratadas con extracto presentaron menor altura y hubo disminución de la biomasa acumulada. De igual modo, la aplicación del extracto al follaje de las malezas redujo la altura y la biomasa acumulada y llegó a causar la muerte de C. intybus, S. pumila y D. carota.
\end{abstract}

Palabras-clave: Convolvulus arvensis, Daucus carota, Setaria pumila, Cichorium intybus, alelopatía.

\begin{abstract}
The objective of this research was to determine the phytotoxic effect of the $n$-hexane extract obtained from the bark of Drimys winteri on germination and growth of Convolvulus arvensis, Setaria pumila, Daucus carota and Cichorium intybus. The phytotoxic effects of the extract on the germination of weeds were determined by bioassays in Petri dishes, adding the extract on the germination medium at concentrations of 100 to $1,000 \mathrm{mg} \mathrm{L}^{-1}$. The phytotoxic effects of the extract on the emergence and seedling growth of weeds were studied in pots bioassays, where the extract was applied mixed with the growth medium at concentrations of 500 to $4,000 \mathrm{mg} \mathrm{kg}^{-1}$. We also determined the phytotoxic effects of the extract by spraying on weed plants, at concentrations of 800 to $6,400 \mathrm{mg} \mathrm{L}^{-1}$. In germination tests, the extract delayed germination and decreased the shoot and radicle growth of all weeds, germination decreased in all weeds except $\boldsymbol{C}$. arvensis. The application of the extract to the growth medium decreased and delayed the emergence of the weeds, plants treated with the extract had lower height and reduced accumulated biomass. The extract sprayed on plants delayed the growth of the four weed species and decreased their cumulative biomass, plant mortality was observed with the highest concentration tested for S. pumila, D. carota and $\mathbf{C}$. intybus.
\end{abstract}

Keywords: Convolvulus arvensis, Daucus carota, Setaria pumila, Cichorium intybus, allelopathy.

1 Recebido para publicação em 14.10.2010 e na forma revisada em 6.5.2011.

2 Profesores, Departamento Producción Vegetal, Facultad de Agronomía, Universidad de Concepción - UDEC, Av. Vicente Méndez 595, Chillán, Chile, <nzapata@udec.cl.>; ${ }^{3}$ Ingeniero Agrónomo, UDEC. 


\section{INTRODUCCIÓN}

Una de las causas de las mayores pérdidas de rendimiento en la agricultura es la constante competencia que ejercen las malezas con los cultivos. Las malezas compiten con los cultivos por agua, luz y nutrientes y destacan por su alta productividad y por la longevidad de sus semillas y por un rápido crecimiento. Por ello, su control es un factor muy importante a tener en cuenta en los sistemas de producción agricola.

Entre las especies de malezas presentes en Chile que causan los mayores daños se encuentran las dicotiledóneas Convolvulus arvensis (Convolvulaceae), Cichorium intybus (Asteraceae) y Daucus carota (Apiaceae). Entre las malezas monocotiledóneas de mayor importancia económica se encuentra Setaria pumila Poiret (Poaceae) (Matthei, 1995).

Durante el proceso de intensificación de la agricultura en las décadas pasadas, los herbicidas organosintéticos han jugado un papel clave en el combate contra las malezas como el método más empleado para su control en la agricultura tecnificada. Sin embargo, debido a la aparición de resistencia por parte de las malezas y al daño que ocasionan los herbicidas al medioambiente, aumenta cada vez más la preocupación y la presión para que se reduzca o elimine el empleo de estas sustancias en la producción de alimentos (Duke et al., 2002). En este contexto, se hace imprescindible explorar otras alternativas menos agresivas con relación al medioambiente para el control de las malezas.

En la búsqueda de alternativas a los herbicidas organosintéticos, las mismas plantas se presentan como una de las fuentes más accesibles y prometedoras, ya que durante toda su existencia, para sobrevivir, han tenido que interaccionar con sus congéneres mediante diversos compuestos químicos, así lo confirman los numerosos y diversos metabolitos aislados de las plantas que han presentado actividad alelopática frente a otras plantas (Rice, 1984; Morita et al., 2005). Se puede aprovechar la presencia de compuestos aleloquímicos en las plantas para el control de malezas en los cultivos, sobre todo en aquellos sistemas productivos que se desarrollan bajo certificación orgánica en los que no se permite el empleo de herbicidas convencionales. La utilización de estas especies puede involucrar el empleo de la vegetación como mulch (Petersen et al., 2001; Rathinasabapathi et al., 2005), la incorporación directa del tejido vegetal al suelo (Sarwar \& Kirkegaard, 1998) o el empleo de extractos con diversos grados de purificación o procesamiento (Orozco \& Tirado, 2004; Kadioglu \& Yanar, 2004; Batish et al., 2004). Por lo general, la forma de utilización dependerá del tipo y disponibilidad de vegetación y de la conveniencia económica.

Drimys winteri (Forster \& Forster) (Winteraceae) es una especie vegetal que presenta gran potencial alelopático. Estudios fitoquímicos indican que sus hojas y corteza presentan altos contenidos de taninos, ácido ascórbico, flavonoides y aceite esencial (Muñoz et al., 1999; Barrero et al., 2000). De la corteza de esta especie también se han aislado y caracterizado numerosos aleloquímicos, principalmente sesquiterpenos del tipo drimano (Cortés et al., 1982; Rodríguez et al., 2005). Este metabolito ha demostrado tener importantes propiedades fitotóxicas (Jansen \& Groot, 2004).

Drimys winteri es una especie arbórea que puede alcanzar hasta $30 \mathrm{~m}$ de altura. Su tronco presenta una corteza de color gris claro, gruesa y blanda. (Hoffmann et al., 1992). En Chile, se puede encontrarla desde la Región de Coquimbo hasta Punta Arenas y es particularmente abundante en la isla de Chiloé. Sólo en la Región de Los Lagos cubre unas 650 mil hectáreas (INFOR, 2004). Esta especie es un recurso forestal nativo muy apreciado por su madera de excelente calidad para la construcción de viviendas y adecuada para la producción de celulosa (Franco et al., 2005). Su explotación forestal genera abundantes residuos, tales como corteza, ramas y hojas, que actualmente no son aprovechados, ya que no son aptos para ser utilizados como combustibles por el humo irritante que producen. Estos residuos podrian emplearse como materia prima para la obtención de extractos con propiedades fitotóxicas y también utilizarse como herbicidas naturales, si se considera la gran cantidad y diversidad de metabolitos secundarios que contiene esta especie (Muñoz et al., 1999; Rodríguez et al., 2005). 
Con el fin de proporcionar antecedentes sobre las propiedades alelopáticas de D. winteri, el objetivo de esta investigación fue determinar el efecto fitotóxico de un extracto obtenido con $\mathrm{n}$-hexano a partir de la corteza de $D$. winteri sobre la germinación y el crecimiento de Convolvulus arvensis, Setaria pumila, Daucus carota y Cichorium intybus.

\section{MATERIALES Y MÉTODOS}

\section{Recolección de corteza y obtención de extractos}

Para obtener los extractos, se recolectó corteza de árboles adultos de la especie $D$. winteri en marzo de 2009 en la comuna de San Ignacio, Región del Bio Bio, Chile. La corteza recolectada fue lavada, secada en horno a $35{ }^{\circ} \mathrm{C}$ durante 72 horas y triturada hasta que se obtuviera partículas de $5 \mathrm{~mm}$ de diámetro. Posteriormente, la corteza (100 g) fue sometida a un proceso de extracción por $48 \mathrm{~h}$ con $\mathrm{n}$-hexano $(2 \times 500 \mathrm{~mL})$. Para separar el solvente del extracto, se empleó rotavapor conectado a bomba de vacío (Büchi $\left.{ }^{\circledR}, \mathrm{R}-210 \mathrm{~A}\right)$. Con este método de extracción se obtuvo $36.12 \mathrm{~g}$ de un extracto de color amarillento de densidad similar a la miel. E1 extracto fue almacenado a $4{ }^{\circ} \mathrm{C}$ hasta su empleo en la experimentación.

\section{Semillas de malezas}

Para este estudio, se utilizaron semillas de las malezas $C$. arvensis, $D$. carota, $S$. pumila y $C$. intybus, las cuales fueron recolectadas en enero de 2009 en la Estación Experimental E1 Nogal de la Universidad de Concepción, Chillán, Chile. Las semillas fueron seleccionadas y desinfectadas con hipoclorito de sodio al $2 \%$ durante 10 minutos. En pruebas de germinación, todas las especies presentaron $>95 \%$ de germinación. C. arvensis fue escarificada mecánicamente antes de a su empleo en la experimentación.

\section{Bioensayos de germinación}

Para conocer el efecto de los extractos sobre la germinación de cada especie de maleza, se establecieron experimentos en placas de Petri $(9 \mathrm{~cm} \varnothing)$ y sobre papel filtro
(Whatman ${ }^{\circledR}$ \# 2). Los extractos se diluyeron en agua hasta alcanzar concentraciones de 100, 250, 500 y $1.000 \mathrm{mg} \mathrm{L}^{-1}$. Para su dilución en agua, los extractos se formularon al $50 \%$ con una mezcla de $\operatorname{Span}^{\circledR} 80$ y Tween ${ }^{\circledR} 20$ (1:3). Sobre el papel filtro de cada placa se aplicaron $3 \mathrm{ml}$ de las soluciones y luego se sembraron 20 semillas de la maleza respectiva. Fueron realizadas cuatro repeticiones para cada concentración y también se incluyó un tratamiento de control en el que se aplicó solamente una solución de emulgentes en una concentración de $0,1 \%$. Durante toda la experimentación, se mantuvo las placas en cámara de germinación sin luz y a una temperatura de $21 \pm 2{ }^{\circ} \mathrm{C}$. Diariamente se contabilizó el número de semillas germinadas. Con los datos obtenidos se determinó el porcentaje de germinación y el índice de velocidad de germinación (Maguire, 1962). También se midió longitud de radícula y tallo de plántula en cada repetición.

\section{Bioensayos en sustrato de cultivo}

Para determinar el efecto fitotóxico del extracto sobre la emergencia y crecimiento de cada especie de maleza, se establecieron ensayos en macetas bajo condiciones de invernadero. Como sustrato de cultivo se empleó suelo franco mezclado con arena en proporción de 9:1, al cual se añadió extracto a una razón de 500, 1.000, 2.000 y $4.000 \mathrm{mg} \mathrm{kg}^{-1}$. Para mezclar homogéneamente el extracto con el sustrato de cultivo, el extracto fue previamente formulado con diatomita $(40 \%$ extracto, $60 \%$ diatomita). Se depositaron $300 \mathrm{~g}$ de sustrato mezclado con extracto en cada maceta, posteriormente se regó hasta saturación y después de 24 horas se procedió la siembra de 20 semillas de la especie de maleza correspondiente. Fueron hechas cuatro repeticiones para cada concentración, y también se incluyó un tratamiento de control en el que se aplicó únicamente diatomita al $0,6 \%$. Los experimentos fueron regados cada dos días de manera homogénea durante todo el período experimental para mantener la humedad del suelo a $60 \%$ de Capacidad de Campo. Diariamente y durante 10 dias se contabilizó el número de plantas emergidas y con los datos obtenidos se calculó porcentaje y velocidad de emergencia. Transcurridos 
21 días desde el 50\% de emergencia en el control, se determinó en cada repetición la altura y la biomasa acumulada por cada planta.

\section{Bioensayos de aspersión de extracto sobre plantas}

Con el fin de determinar el efecto fitotóxico del extracto aplicado al follaje de las malezas se establecieron experimentos en macetas bajo condiciones de invernadero. Se sembraron 20 semillas de cada especie de maleza por maceta y se empleó como sustrato suelo franco. En etapa de tres a cuatro hojas se efectuó un raleo, dejando sólo 10 plantas por maceta, que posteriormente fueron tratadas con el extracto. Se prepararon soluciones de extracto en concentraciones de 800, 1.600, 3.200, $6.400 \mathrm{mg} \mathrm{L}^{-1}$, las cuales fueron formuladas de igual modo a lo descrito con relación a los bioensayos de germinación. Las plantas de cada maceta fueron asperjadas por una única vez con $10 \mathrm{~mL}$ de soluciones de extracto con un aspersor manual posicionado a $20 \mathrm{~cm}$ de distancia de las plantas. El volumen de solución aplicada logró mojar las plantas hasta el punto de goteo. Se establecieron cuatro repeticiones para cada concentración y un tratamiento de control en el que se aplicó solamente una solución de emulgentes a una concentración de $0,1 \%$. En cada repetición se midió la altura de planta, previamente a aplicación del extracto y posteriormente a los dias 7, 14 y 21 desde la aplicación. Transcurridos 21 días de la aplicación, se determinó la biomasa acumulada por planta. También se realizaron observaciones de daño visual transcurridos 2 , 7, 14 y 21 días de la aplicación del extracto. La fitotoxicidad se evaluó mediante la escala propuesta por ALAM (1974), la cual se basa en el nivel de daño visual que se observa en plantas por efecto de la aplicación de un herbicida. La escala va de cero (0), correspondiente a ningún daño, hasta diez (10), que corresponde a la muerte total de la planta.

\section{Diseño experimental y análisis de datos}

Para los experimentos se empleó un diseño completamente al azar. Los datos obtenidos fueron sometidos a análisis de varianza (ANDEVA) y sus medias fueron comparadas mediante test DMS $(p \leq 0,05)$. En los experimentos de aspersión sobre plantas, para el análisis estadístico de las alturas de planta, se empleó como covariable la altura de planta previa a la aplicación del extracto. Para efectuar los análisis estadísticos, se utilizó el software Statgraphics ${ }^{\circledR}$ Plus Versión 5.0 (STSC, 1987).

\section{RESULTADOS Y DISCUSIÓN}

Los resultados obtenidos en este estudio sobre la actividad fitotóxica del extracto obtenido con $\mathrm{n}$-hexano a partir de la corteza de $D$. winteri en malezas de importancia económica en Chile son muy promisorios, indican que la corteza de este árbol presenta metabolitos secundarios con elevada actividad fitotóxica y que es posible extraerlos con solventes orgánicos de baja polaridad.

\section{Bioensayos de germinación}

A excepción de $C$. arvensis, la aplicación de extracto $\mathrm{n}$-hexano de $D$. winteri en el medio de germinación disminuyó significativamente la germinación de las malezas en estudio, incluso al aplicarse la menor dosis de extracto (100 $\mathrm{mg} \mathrm{L}^{-1}$ ) (Tabla 1). Aún cuando el extracto no afectó el porcentaje de germinación de $C$. arvensis, se retardó significativamente su velocidad de germinación a contar de la concentración de $500 \mathrm{mg} \mathrm{L}^{-1}$. La longitud de radícula y de tallo de esta especie disminuyeron en más de un $90 \%$ con relación al control, lo cual indica que, si bien no se produjo disminución en el número de semillas germinadas, el extracto sí detuvo el crecimiento de las plántulas. En comparación con el control, C. intybus, S. pumila y D. carota mostraron una menor velocidad de germinación y crecimiento posterior de las plántulas, incluso con la concentración más baja de extracto aplicada al medio de germinación $\left(100 \mathrm{mg} \mathrm{L}^{-1}\right)$, lo cual denota la alta sensibilidad de estas especies de malezas al extracto evaluado.

La actividad fitótoxica de los extractos evaluados resultó dependiente de la concentración aplicada al medio de germinación, aunque su efecto también dependa de la especie de maleza de que se trate, de modo general, al ordenar las especies de mayor a menor sensibilidad al extracto, tenemos $S$. pumila $>D$. carota $>C$. intybus $>C$. arvensis. 
Tabla 1 - Efecto de un extracto n-hexano obtenido de la corteza de $D$. winteri sobre la germinación y el crecimiento de cuatro especies de malezas

\begin{tabular}{|c|c|c|c|c|c|}
\hline \multirow{2}{*}{$\begin{array}{l}\text { Especie de } \\
\text { maleza }\end{array}$} & \multirow{2}{*}{$\frac{\text { Extracto }}{\left(\mathrm{mg} \mathrm{L}^{-1}\right)}$} & \multirow{2}{*}{$\frac{\text { Germinación }}{(\%)}$} & \multirow{2}{*}{$\frac{\mathrm{VG}}{\left(\text { Planta día }^{-1}\right)}$} & \multicolumn{2}{|c|}{ Longitud (mm) } \\
\hline & & & & Radícula & Tallo \\
\hline \multirow{5}{*}{ C. arvensis } & 0 & $100,00 \mathrm{a}$ & $17,46 \mathrm{a}$ & $27,50 \mathrm{a}$ & $35,10 \mathrm{a}$ \\
\hline & 100 & $100,00 \mathrm{a}$ & $15,85 \mathrm{ab}$ & $24,85 \mathrm{~b}$ & $30,05 \mathrm{~b}$ \\
\hline & 250 & $100,00 \mathrm{a}$ & $15,65 \mathrm{ab}$ & $10,90 \mathrm{c}$ & $18,40 \mathrm{c}$ \\
\hline & 500 & $100,00 \mathrm{a}$ & $14,94 \mathrm{~b}$ & $8,40 \mathrm{~d}$ & $12,10 \mathrm{~d}$ \\
\hline & 1.000 & $98,75 \mathrm{a}$ & $10,50 \mathrm{c}$ & $2,60 \mathrm{e}$ & $2,90 \mathrm{e}$ \\
\hline $\mathrm{CV}(\%)$ & & 1,12 & 8,33 & 8,22 & 11,59 \\
\hline \multirow{5}{*}{ C. intybus } & 0 & $97,50 \mathrm{a}$ & $8,43 \mathrm{a}$ & $26,70 \mathrm{a}$ & $32,25 \mathrm{a}$ \\
\hline & 100 & $66,25 \mathrm{~b}$ & $5,81 \mathrm{~b}$ & $21,70 \mathrm{~b}$ & $24,80 \mathrm{~b}$ \\
\hline & 250 & $60,00 \mathrm{~b}$ & $3,83 \mathrm{c}$ & $12,60 \mathrm{c}$ & $18,85 \mathrm{c}$ \\
\hline & 500 & $51,25 \mathrm{c}$ & $3,56 \mathrm{c}$ & $10,50 \mathrm{c}$ & $16,05 \mathrm{c}$ \\
\hline & 1.000 & $45,00 \mathrm{c}$ & $2,70 \mathrm{~d}$ & $6,35 \mathrm{~d}$ & $13,10 \mathrm{~d}$ \\
\hline CV $(\%)$ & & 7,13 & 14,03 & 9,48 & 9,69 \\
\hline \multirow{5}{*}{ S. pumila } & 0 & $100,00 \mathrm{a}$ & $17,19 \mathrm{a}$ & $29,83 \mathrm{a}$ & $56,60 \mathrm{a}$ \\
\hline & 100 & $85,06 \mathrm{~b}$ & $13,03 \mathrm{~b}$ & $24,93 \mathrm{~b}$ & $29,25 \mathrm{~b}$ \\
\hline & 250 & $75,00 \mathrm{~b}$ & $3,68 \mathrm{c}$ & $7,90 \mathrm{c}$ & $19,20 \mathrm{c}$ \\
\hline & 500 & $26,25 \mathrm{c}$ & $1,49 \mathrm{~d}$ & $7,25 \mathrm{c}$ & $0,55 \mathrm{~d}$ \\
\hline & 1.000 & $15,00 \mathrm{~d}$ & $0,70 \mathrm{~d}$ & $2,20 \mathrm{~d}$ & $0,00 \mathrm{~d}$ \\
\hline CV (\%) & & 7,29 & 14,83 & 10,5 & 4,86 \\
\hline \multirow{5}{*}{ D. carota } & 0 & $98,75 \mathrm{a}$ & $6,41 \mathrm{a}$ & $27,50 \mathrm{a}$ & $35,10 \mathrm{a}$ \\
\hline & 100 & $80,00 \mathrm{~b}$ & $4,82 \mathrm{~b}$ & $21,13 \mathrm{~b}$ & $30,05 \mathrm{~b}$ \\
\hline & 250 & $76,25 \mathrm{~b}$ & $3,31 \mathrm{c}$ & $9,95 \mathrm{c}$ & $19,14 \mathrm{c}$ \\
\hline & 500 & $68,75 \mathrm{~b}$ & $2,78 \mathrm{c}$ & $7,65 \mathrm{~d}$ & $12,10 \mathrm{~d}$ \\
\hline & 1.000 & $10,00 \mathrm{c}$ & $0,33 \mathrm{~d}$ & $2,90 \mathrm{e}$ & $2,35 \mathrm{e}$ \\
\hline CV $(\%)$ & & 14,51 & 18,2 & 7,32 & 11,4 \\
\hline
\end{tabular}

Medias seguidas de letras iguales, en la misma columna, no difieren estadísticamente entre si según test DMS ( $p \leq 0,05)$. VG: Velocidad de germinación. CV: Coeficiente de variación

Independiente de la especie de maleza, para las mayores concentraciones de extracto en el medio de germinación (500 y $1.000 \mathrm{mg} \mathrm{L}^{-1}$ ), se observaron plántulas anormales con raíces primarias deformes y tallos gruesos con relación al control (datos no mostrados). Este comportamiento es similar al producido por los herbicidas del tipo reguladores de crecimiento, los cuales atrofian el crecimiento y desarrollo de la plántula, cuando no lo inhiben completamente (Kogan \& Pérez, 2003).

\section{Bioensayos en sustrato de cultivo}

El extracto adicionado al sustrato de cultivo redujo significativamente la emergencia de las cuatro especies de malezas y afectó el crecimiento posterior de las plántulas (Tabla 2).
Las especies C. arvensis y S. pumila mostraron un mayor grado de tolerancia al extracto, considerando que su emergencia sólo disminuyó significativamente respecto al control cuando se trató el sustrato de cultivo con extracto a una razón de $2.000 \mathrm{mg} \mathrm{kg}{ }^{-1}$. En cambio, $C$. intybus y $D$. carota demostraron ser más sensibles al extracto y no registraron emergencia cuando sembradas en sustrato de cultivo tratado con $4.000 \mathrm{mg} \mathrm{kg}^{-1}$ de extracto. La velocidad de emergencia de las malezas también se redujo a partir de la menor concentración de extracto aplicada, excepto para C. arvensis que sólo disminuyó en las dos mayores concentraciones aplicadas. El extracto n-hexano también afectó el crecimiento posterior de las plántulas, incluso a partir de la menor dosis evaluada (500 $\mathrm{mg} \mathrm{kg}^{-1}$ ). La 
Tabla 2 - Efecto de un extracto n-hexano obtenido de la corteza de D. winteri aplicado en el sustrato de cultivo sobre la emergencia y el crecimiento de cuatro especies de malezas

\begin{tabular}{|c|c|c|c|c|c|}
\hline \multirow{2}{*}{ Especie de maleza } & Extracto & Emergencia & $\mathrm{VE}$ & Altura total & Biomasa \\
\hline & $\left(\mathrm{mg} \mathrm{kg}^{-1}\right)$ & $(\%)$ & $\left(\right.$ Planta día $\left.^{-1}\right)$ & $(\mathrm{mm})$ & (mg por planta) \\
\hline \multirow{5}{*}{ C. arvensis } & 0 & $100,00 \mathrm{a}$ & $7,95 \mathrm{a}$ & $39,25 \mathrm{a}$ & $217,50 \mathrm{a}$ \\
\hline & 500 & $100,00 \mathrm{a}$ & $7,01 \mathrm{a}$ & $29,00 \mathrm{~b}$ & $152,50 \mathrm{~b}$ \\
\hline & 1.000 & $100,00 \mathrm{a}$ & $6,73 \mathrm{ab}$ & $22,75 \mathrm{c}$ & $125,00 \mathrm{~b}$ \\
\hline & 2.000 & $45,00 \mathrm{~b}$ & $5,22 \mathrm{~b}$ & $15,75 \mathrm{~d}$ & $100,00 \mathrm{bc}$ \\
\hline & 4.000 & $42,50 \mathrm{~b}$ & $3,45 \mathrm{c}$ & $4,00 \mathrm{e}$ & $67,50 \mathrm{c}$ \\
\hline $\mathrm{CV}(\%)$ & & 6,45 & 16,92 & 18,07 & 29,89 \\
\hline \multirow{5}{*}{ C. intybus } & 0 & $100,00 \mathrm{a}$ & $5,14 \mathrm{a}$ & $34,25 \mathrm{a}$ & $177,50 \mathrm{a}$ \\
\hline & 500 & $47,50 \mathrm{~b}$ & $0,68 \mathrm{~b}$ & $25,00 \mathrm{~b}$ & $140,00 \mathrm{~b}$ \\
\hline & 1.000 & $12,50 \mathrm{c}$ & $0,14 \mathrm{c}$ & $18,50 \mathrm{~b}$ & $105,00 \mathrm{c}$ \\
\hline & 2.000 & $2,50 \mathrm{~d}$ & $0,03 \mathrm{c}$ & $2,00 \mathrm{c}$ & $12,50 \mathrm{~d}$ \\
\hline & 4.000 & $0,00 \mathrm{~d}$ & $0,00 \mathrm{c}$ & $0,00 \mathrm{c}$ & $0,00 \mathrm{~d}$ \\
\hline CV $(\%)$ & & 11,92 & 44,25 & 30,36 & 22,7 \\
\hline \multirow{5}{*}{ S. pumila } & 0 & $100,00 \mathrm{a}$ & $7,46 \mathrm{a}$ & $40,50 \mathrm{a}$ & $155,00 \mathrm{a}$ \\
\hline & 500 & $100,00 \mathrm{a}$ & $5,18 \mathrm{~b}$ & $29,50 \mathrm{~b}$ & $102,50 \mathrm{~b}$ \\
\hline & 1.000 & $100,00 \mathrm{a}$ & $3,94 \mathrm{c}$ & $26,25 \mathrm{~b}$ & $67,50 \mathrm{c}$ \\
\hline & 2.000 & $90,00 \mathrm{~b}$ & $2,61 \mathrm{~d}$ & $18,50 \mathrm{c}$ & $45,00 \mathrm{c}$ \\
\hline & 4.000 & $52,50 \mathrm{c}$ & $1,65 \mathrm{e}$ & $7,25 \mathrm{~d}$ & $42,50 \mathrm{c}$ \\
\hline $\mathrm{CV}(\%)$ & & 6,36 & 13,28 & 17,46 & 20,34 \\
\hline \multirow{5}{*}{ D. carota } & 0 & $100,00 \mathrm{a}$ & $6,01 \mathrm{a}$ & $33,25 \mathrm{a}$ & $182,50 \mathrm{a}$ \\
\hline & 500 & $100,00 \mathrm{a}$ & $4,57 \mathrm{~b}$ & $25,75 \mathrm{~b}$ & $145,00 \mathrm{~b}$ \\
\hline & 1.000 & $87,50 \mathrm{~b}$ & $3,12 \mathrm{c}$ & $23,25 \mathrm{~b}$ & $117,50 \mathrm{c}$ \\
\hline & 2.000 & $40,00 \mathrm{c}$ & $1,13 \mathrm{~d}$ & $12,25 \mathrm{c}$ & $75,00 \mathrm{~d}$ \\
\hline & 4.000 & $0,00 \mathrm{~d}$ & $0,00 \mathrm{e}$ & $0,00 \mathrm{~d}$ & $0,00 \mathrm{e}$ \\
\hline $\mathrm{CV}(\%)$ & & 8,59 & 17,15 & 20,12 & 13,93 \\
\hline
\end{tabular}

Medias seguidas de letras iguales, en la misma columna, no difieren estadísticamente entre si según test DMS (p $\leq 0,05)$. VE: Velocidad de emergencia. $\mathrm{CV}$ : Coeficiente de variación.

altura de planta y la biomasa total acumulada disminuyeron significativamente con el incremento de la dosis de extracto aplicada en todas las malezas.

La menor capacidad de crecimiento expresado como altura y biomasa acumulada que presentaron las malezas tratadas con dosis crecientes de extracto es un indicativo de efecto fitotóxico residual del extracto prolongado en el tiempo. Esto puede deberse a que este tipo de extractos es una mezcla compleja de diversos metabolitos (Rodríguez et al., 2005) con conocida actividad antimicrobiana (Monsálvez et al., 2010), por lo cual su degradación en el suelo es muy lenta. E1 efecto fitotóxico prolongado de este extracto aplicado al suelo podría representar una ventaja importante para algunas aplicaciones prácticas como desinfección de sustratos de cultivo.

\section{Bioensayos de aspersión de extracto sobre plantas}

Este extracto también demostró tener acción fitotóxica muy potente cuando se lo aplicó por aspersión sobre el follaje de las malezas (Tablas 3 y 4). Transcurridos 7 días de la aplicación, la altura de la planta de las malezas disminuyó significativamente respecto al control, incluso con la menor dosis de extracto aplicada (800 $\left.\mathrm{mg} \mathrm{L}^{-1}\right)$; igual tendencia se observó transcurridos 14 y 21 días de la aplicación. El extracto asperjado al follaje de las malezas resultó en una menor biomasa 
acumulada por planta, dependiendo de la dosis aplicada. Esta respuesta estuvo de acuerdo con lo señalado previamente para la altura de planta. La biomasa acumulada por planta representó aproximadamente la tercera parte de la biomasa acumulada en el control para C. intybus, D. carota y S. pumila, a la máxima concentración de extracto aplicada. A su vez, para $C$. arvensis la biomasa acumulada alcanzó al $57 \%$ de la obtenida en el control.

Las observaciones visuales que se presentan en la Tabla 4, de acuerdo con la escala de la ALAM, indican que al segundo día tras efectuarse la aspersión C. arvensis presentó sólo daño leve (índice 1) a partir de la aplicación de extracto a concentración de $1.600 \mathrm{mg} \mathrm{L}^{-1}$. A su vez, C. intybus, S. pumilay
D. carota presentaron daño de leve a moderado (indice 2) a partir de la concentración $800 \mathrm{mg} \mathrm{L}^{-1}$. El índice de daño se incrementó con el tiempo y particularmente para las mayores concentraciones aplicadas. Transcurridos 21 días desde la aplicación, para la máxima concentración de extracto aplicada, se observó un nivel de daño severo (indice 4) para C. arvensis y $C$. intybus, nivel de daño muy severo (índice 5) para $S$. pumila y daño grave (indice 6) para $D$. carota. Se observaron plantas muertas en los tratamientos de mayor concentración (un $10 \%$ en $C$. intybus, un $60 \%$ en S. pumila y un $50 \%$ en $D$. carota).

En términos generales, el extracto aplicado por aspersión sobre las malezas mostró actividad fitotóxica similar para todas las especies,

Tabla 3 - Efecto de un extracto n-hexano obtenido de la corteza de $D$. winteri aplicado por aspersión sobre el follaje en el crecimiento de cuatro especies de malezas

\begin{tabular}{|c|c|c|c|c|c|}
\hline \multirow{2}{*}{ Especie de maleza } & \multirow{2}{*}{$\begin{array}{l}\text { Extracto } \\
\left(\mathrm{mg} \mathrm{L}^{-1}\right)\end{array}$} & \multicolumn{3}{|c|}{ Altura a diferentes días tras aplicación (mm) } & \multirow{2}{*}{$\frac{\text { Biomasa }}{(\mathrm{mg} \text { por planta) }}$} \\
\hline & & Día 7 & Día 14 & Día 21 & \\
\hline \multirow{5}{*}{ C. arvensis } & 0 & $35,74 \mathrm{a}$ & $40,62 \mathrm{a}$ & $48,41 \mathrm{a}$ & $50,83 \mathrm{a}$ \\
\hline & 800 & $32,54 \mathrm{~b}$ & $34,60 \mathrm{~b}$ & $38,80 \mathrm{~b}$ & $40,00 \mathrm{~b}$ \\
\hline & 1.600 & $32,01 \mathrm{~b}$ & $35,95 \mathrm{~b}$ & $36,96 \mathrm{~b}$ & $31,67 \mathrm{c}$ \\
\hline & 3.200 & $31,46 \mathrm{~b}$ & $33,24 \mathrm{~b}$ & $35,85 \mathrm{~b}$ & $25,00 \mathrm{~d}$ \\
\hline & 6.400 & $25,51 \mathrm{c}$ & $30,09 \mathrm{c}$ & $31,73 \mathrm{c}$ & $28,83 \mathrm{~d}$ \\
\hline $\mathrm{CV}(\%)$ & & 3,94 & 5,57 & 9,23 & 11,46 \\
\hline \multirow{5}{*}{ C. intybus } & 0 & $27,00 \mathrm{a}$ & $29,10 \mathrm{a}$ & $32,37 \mathrm{a}$ & $64,50 \mathrm{a}$ \\
\hline & 800 & $25,04 \mathrm{~b}$ & $25,83 \mathrm{~b}$ & $27,33 \mathrm{~b}$ & $48,67 \mathrm{~b}$ \\
\hline & 1.600 & $25,00 \mathrm{~b}$ & $25,36 \mathrm{~b}$ & $28,84 \mathrm{~b}$ & $38,67 \mathrm{c}$ \\
\hline & 3.200 & $22,13 b c$ & $21,25 \mathrm{c}$ & $23,97 \mathrm{c}$ & $28,67 \mathrm{~d}$ \\
\hline & 6.400 & $18,57 \mathrm{c}$ & $18,46 \mathrm{c}$ & $22,49 \mathrm{c}$ & $17,00 \mathrm{e}$ \\
\hline $\mathrm{CV}(\%)$ & & 6,79 & 9,43 & 6,84 & 8,08 \\
\hline \multirow{5}{*}{ S. pumila } & 0 & $84,87 \mathrm{a}$ & $96,90 \mathrm{a}$ & $103,25 \mathrm{a}$ & $64,17 \mathrm{a}$ \\
\hline & 800 & $74,90 \mathrm{~b}$ & $81,62 \mathrm{~b}$ & $88,30 \mathrm{~b}$ & $49,17 \mathrm{~b}$ \\
\hline & 1.600 & $73,69 \mathrm{~b}$ & $76,46 \mathrm{bc}$ & $82,00 \mathrm{bc}$ & $36,67 \mathrm{c}$ \\
\hline & 3.200 & $72,16 \mathrm{~b}$ & $74,63 \mathrm{c}$ & $77,39 \mathrm{c}$ & $27,50 \mathrm{~d}$ \\
\hline & 6.400 & $71,37 b$ & $73,89 \mathrm{c}$ & $75,56 \mathrm{c}$ & $22,50 \mathrm{e}$ \\
\hline $\mathrm{CV}(\%)$ & & 3,6 & 6,21 & 7,16 & 7,76 \\
\hline \multirow{5}{*}{ D. carota } & 0 & $84,67 \mathrm{a}$ & $98,07 \mathrm{a}$ & $106,65 \mathrm{a}$ & $65,00 \mathrm{a}$ \\
\hline & 800 & $59,02 \mathrm{~b}$ & $65,55 \mathrm{~b}$ & $69,50 \mathrm{~b}$ & $47,50 \mathrm{~b}$ \\
\hline & 1.600 & $47,04 \mathrm{c}$ & $52,04 \mathrm{c}$ & $61,34 \mathrm{~b}$ & $35,83 \mathrm{c}$ \\
\hline & 3.200 & $35,82 \mathrm{~d}$ & $45,69 \mathrm{c}$ & $49,41 \mathrm{c}$ & $29,17 \mathrm{~d}$ \\
\hline & 6.400 & $32,21 \mathrm{~d}$ & $31,41 \mathrm{~d}$ & $31,85 \mathrm{~d}$ & $21,67 \mathrm{e}$ \\
\hline $\mathrm{CV}(\%)$ & & 7,31 & 11,95 & 13,75 & 8,16 \\
\hline
\end{tabular}

Medias seguidas de letras iguales, en la misma columna, no difieren estadísticamente entre si según test DMS ( $\leq \leq 0,05)$. CV: Coefíciente de variación. 
Tabla 4 - Evaluación visual de fitotoxicidad de extracto n-hexano obtenido de la corteza de $D$. winteri aplicado por aspersión sobre el follaje de cuatro especies de malezas

\begin{tabular}{|c|c|c|c|c|c|}
\hline \multirow{2}{*}{$\begin{array}{c}\text { Especie de } \\
\text { maleza }\end{array}$} & $\begin{array}{c}\text { Concentración } \\
\text { extracto }\end{array}$ & \multicolumn{4}{|c|}{$\begin{array}{c}\text { Índice de fitotoxicidad }{ }^{1 /} \text {, días } \\
\text { tras aplicación }\end{array}$} \\
\cline { 2 - 6 } & $\left(\mathrm{mg} \mathrm{L}^{-1}\right)$ & Día 2 & Día 7 & Día 14 & Día 21 \\
\hline & 0 & 0 & 0 & 0 & 0 \\
\cline { 2 - 6 } C. arvensis & 800 & 0 & 1 & 1 & 1 \\
\cline { 2 - 6 } & 1.600 & 1 & 2 & 2 & 2 \\
\cline { 2 - 6 } & 3.200 & 1 & 2 & 3 & 3 \\
\cline { 2 - 6 } & 6.400 & 1 & 3 & 4 & 4 \\
\cline { 2 - 6 } & 0 & 0 & 0 & 0 & 0 \\
\cline { 2 - 6 } & 1.600 & 2 & 2 & 2 & 2 \\
\cline { 2 - 6 } & 3.200 & 2 & 2 & 2 & 4 \\
\cline { 2 - 6 } & 6.400 & 2 & 2 & 2 & 4 \\
\hline \multirow{5}{*}{ S. pumtybus } & 0 & 0 & 0 & 0 & 0 \\
\cline { 2 - 6 } & 800 & 1 & 1 & 2 & 1 \\
\cline { 2 - 6 } & 1.600 & 1 & 2 & 2 & 1 \\
\cline { 2 - 6 } & 3.200 & 2 & 3 & 4 & 4 \\
\cline { 2 - 6 } & 6.400 & 2 & 3 & 4 & 5 \\
\hline \multirow{5}{*}{ D. carota } & 0 & 0 & 0 & 0 & 0 \\
\cline { 2 - 6 } & 1.600 & 2 & 3 & 3 & 5 \\
\cline { 2 - 6 } & 3.200 & 2 & 3 & 4 & 6 \\
\cline { 2 - 6 } & 6.400 & 2 & 3 & 4 & 6 \\
\hline
\end{tabular}

1/ Según escala ALAM.

es decir, presentó escasa selectividad. Esto no debería sorprender si se considera que este extracto es una mezcla compleja de metabolitos que incluso podrian presentar sinergias entre ellos (Philogène, 2003). Es conocido que entre los compuestos de baja polaridad más abundantes que están presentes en la corteza de $D$. winteri se encuentran varios sesquiterpenos (Jansen \& Groot, 2004). Este tipo de compuestos posee un amplio espectro de actividad biológica $\mathrm{y}$, al parecer, juegan un rol importante entre los mecanismos de defensa de las plantas (Gershenzon \& Dudareva, 2007). Cuando la corteza de $D$. winteri se sometió a extracción con $n$-hexano se obtuvo e identificó principalmente sesquiterpenos del tipo drimano (Rodríguez et al., 2005; Zapata et al., 2009). Entre estos drimanos, el poligodial es el que se encuentra en mayor concentración en la corteza seca de $D$. winteri $(8,15 \%)$ (Rodríguez et al., 2005).
Una pregunta que se debe responder es si poligodial $\mathrm{u}$ otros sesquiterpenos del tipo drimano presentes en la corteza de $D$ winteri son responsables de la actividad fitotóxica observada. Es evidente que para responder esta interrogante, se debe fraccionar químicamente el extracto obtenido con $\mathrm{n}$-hexano y evaluar la actividad fitotóxica de los compuestos obtenidos. Sin embargo, resultados obtenidos en estudios previos indican que poligodial sería en parte responsable de esta actividad. Medios de germinación tratados con $10 \mu \mathrm{M}$ de poligodial inhibieron el crecimiento de Lactuca sativa y Setaria italica en un 50\% (Jansen \& Groot, 2004). A su vez, concentraciones de $50 \mu \mathrm{g} \mathrm{mL}^{-1}$ de poligodial impidieron completamente la germinación de $L$. sativa y 25,0 $\mu \mathrm{g} \mathrm{mL}^{-1}$ inhibieron la elongación radicular de esta misma especie en un 50\% (Fujita \& Kubo, 2003).

En esta investigación se demuestra la actividad fitotóxica de un extracto obtenido con n-hexano a partir de corteza de $D$. winteri. Este extracto aplicado en concentraciones $\leq 0,1 \%$ reduce la germinación y el crecimiento de las malezas $C$. arvensis, $C$. intybus, S. pumila y D. carota. La aplicación de este extracto mezclado con el sustrato de cultivo en concentraciones $\leq 0,4 \%$ reduce y retarda $1 \mathrm{a}$ emergencia y el crecimiento de todas las especies de malezas en estudio. Asimismo, la aplicación del extracto al follaje de las malezas a concentraciones $\leq 0,64 \%$ disminuye su crecimiento y llega incluso a causar la muerte de $C$. intybus, S. pumilay D. carota.

Los resultados obtenidos en esta investigación indican la conveniencia de investigar más a fondo esta propiedad fitotóxica y dilucidar los metabolitos responsables de su actividad fitotóxica, así como su posible utilización a nivel de campo para el control de malezas en sistemas de producción agrícola.

\section{AGRADECIMIENTOS}

Esta investigación ha sido financiada por Proyecto Fondecyt № 11070060.

\section{LITERATURA CITADA}

ALAM. Métodos para la evaluación de ensayos en control de malezas en Latinoamérica. ALAM, v. 1, n. 1, p. 6-12, 1974. 
BATISH, D. et al. Phytotoxicity of lemon-scented eucalypt oil and its potential use as a bioherbicide. Crop Protec., v. 23, n. 12, p. 1209-1214, 2004.

BARRERO, A. et al. Chemical composition of the essential oil from Drimys winteri Forst. Wood. J. Essent. Oil Res., v. 12, n. 6, p. $685-688,2000$

CORTÉS, M.; URREJOLA, R.; OYARZÚN, M. Metabolitos secundarios de la corteza de Drimys winteri Forster. B. Soc. Chil. Quimica, v. 27, n. 2, p. 307-309, 1982

DUKE, S. et al. Chemicals from nature for weed management. Weed Sci., v. 50, n. 2, p. 138-151, 2002.

FRANCO, H. et al. Kraft pulping of Drimys winteri wood chips biotreated with Ganoderma australe. J. Chem.

Technol. Biotechnol., v. 81, n. 2, p. 196-200, 2005.

FUJTA, K.; KUBO, I. Synergism of polygodial and transcinnamic acid on inhibition of root elongation in lettuce seedling growth bioassays. J. Chem. Ecol., v. 29, n. 10, p. $2253-2262,2003$

GERSHENZON, J.; DUDAREVA, N. The function of terpene natural products in the natural world. Nat. Chem. Biol., v. 3, n. 7, p. 488-414, 2007.

HOFFMANN, A. et al. Plantas medicinales de uso común en Chile. Santiago: Fundación Claudio Gay, 1992. 275 p.

INFOR. Estudios de mercado para la madera de canelo en Estados Unidos y Europa. Santiago: Instituto Forestal, 2004. 92 p. (Informe Técnico, 167)

JANSEN, B.; GROOT, A. Occurrence, biological activity and synthesis of drimane sesquiterpenoids. Nat. Produc. Rep., v. 21, n. 4 , p. $449-477,2004$

KADIOGLU, I.; YANAR, Y. Allelopathic effects of plant extracts against seed germination of some weeds. Asian J. Plant Sci., v. 3, n. 4, p. 472-475, 2004.

KOGAN, M.; PÉREZ, A. Herbicidas: fundamentos fisiológicos y bioquímicos del modo de acción. Santiago: Ediciones Universidad Católica de Chile, 2003. 333 p.

MAGUIRE, J. D. Speed of germination: Aid in selection and evaluation for seedling emergence and vigor. Crop Sci., v. 2, n. 2, p. 176-177, 1962.

MATTHEI, O. Manual de las malezas que crecen en Chile. Santiago: Alfabeta Impresores, 1995. 545 p.
MONSÁLVEZ, M. et al. Antifungal effects of n-hexane extract and essential oil of Drimys winteri bark against Take-All disease. Ind. Crop Prod., v. 31, n. 2, p. 239-244, 2010 .

MORITA, S.; ITO, M.; HARADA, J. Screening of an allelopathic potential in arbor species. Weed Biol. Manag., v. 5, n. 1, p. 26-30, 2005

MUÑOZ, O; MONTES, M.; WILKOMIRSKY, T. Plantas medicinales de uso en Chile. Santiago: Editorial Universitaria., 1999. $330 \mathrm{p}$.

OROZCO, A.; TIRADO, G. Efecto herbicida de extractos vegetales para el manejo de malezas en algodón en el caribe colombiano. Manejo Integr. Plagas, v. 71, n. 5, p. 79-84, 2004.

PETERSEN J. et al. Weed suppression by release of isothiocynates from turnip-rape mulch. Agron. J., v. 93, n. 1, p. $37-43,2001$.

PHILOGÈNE, B. Acción sinérgica de los compuestos de origen vegetal. In: REGNAULTT-ROGER, C.; PHILOGÈNE, B.; VINCENT, C. (Eds.). Biopesticidas de origen vegetal. Madrid: Ediciones Mundi-Prensa, 2003. p. 67-76

RATHINASABAPATHI, B.; FERGUSON, J.; GAL, M. Evaluation of allelopathic potential of wood chips for weed suppression in horticultural production systems. Hort Sci. v. 40, n. 3 , p. $711-713,2005$

RICE, E. L. Allelopathy. 2.ed. New York: Academic Press, 1984. 345 p.

RODRÍGUEZ, B. et al. A complete ${ }^{1} \mathrm{H}$ and ${ }^{13} \mathrm{C}$ NMR data assignment for four drimane sesquiterpenoids isolated from Drimys winterii. Mag. Res. Chem., v. 43, n. 1, p. 82-84, 2005.

SARWAR, M.; KIRKEGAARD, J. Biofumigation potential of brassicas II. Effect of environment and ontogeny on glucosinolate production and implications for screening. Plant Soil, v. 201, n. 1, p. 91-101, 1998.

STSC. User's guide statgraphics. Rockville: Graphic Software System STSC, 1987.

ZAPATA, N. et al. Antifeedant and growth inhibitory effects of extracts and drimanes of Drimys winteri stem bark against Spodoptera littoralis (Lep., Noctuidae). Ind. Crop Prod., v. 30, n. 1, p. $119-125,2009$ 